\title{
Das Metastufenwesen ist das Metasprachenwesen ${ }^{1}$
}

\author{
Hans Lenk \\ https://orcid.org/0000-0003-2910-3671
}

Ioanna Kucuradi nach langjähriger Zusammenarbeit in Freundschaft gewidmet

Menschen haben die Fähigkeit auf höhere Metastufen der Darstellung, Erfassung und Handlung aufzusteigen - und dadurch zu generalisieren und höherstufig zu flexibilisieren. Der Mensch ist also das Metastufenwesen. Das Metastufenwesen kann auch antizipieren und sich auch mit beobachteten anderen Lebewesen imaginativ identifizieren (worauf z. B. Humor, Ironie und Witz beruhen). Der Ansatz vom metainterpretierenden Wesen sollte allgemein auf das Anthropicum „Metastufenwesen” (einen der Metaabstufungen fähigen Symbolgeneralisten) ausgedehnt werden. So vielfältig Eigenschaften, Fähigkeiten und Kennzeichnungen des Menschen auch sein mögen, in allen Bereichen scheinen sie Metastufenbildung zu erlauben. Die intellektuelle motivationale Flexibilität des Menschen ist geradezu gekennzeichnet durch diese seine Fähigkeit, Metastufen zu bilden und in Symbolisierungen und Repräsentation sowie Imagination einzusetzen. Kurz: der Mensch läßt sich als das Metastufenwesen seiner vielfältigen Fähigkeiten und Tätigkeiten sowie Symbolisierungen charakterisieren. Dies ist ein Ansatz, den die Sozial- und Kulturanthropologie empirisch und besonders die philosophische Anthropologie begrifflich, methodologisch und philosophisch weiter verfolgen sollten.

Schlüsselworte: Metastufen-Wesen, Meta-Interpretation, animal symbolicum et metasymbolicum, Anthropologicum, Menschlichkeitskriterium

HANS LENK, professor emeritus, Karlsruhe Institute of Technology, Germany; e-mail: hans.lenk@kit.edu

${ }^{1}$ Es ist eine leicht abgeänderte Fassung des Aufsatzes „Das Metastufenwesen ist das Metasprachenwesen", der früher veröffentlicht war in: S. Iyi (Hg.). Essays in Honor of Ioanna Kucuradi, Sonderband: Journal of Turkish Studies 125, Cambridge, MA, Harvard UP, 2001 [Hans Lenk]. 
Allgemein bekannt ist Cassirers Definition des Menschen als des Animal symbolicum, des Wesens, das eine symbolische Welt, ein „symbolisches Universum“ aufspannt, in dem es gleichsam „lebt“. Der Mensch tritt „der Wirklichkeit nicht mehr unmittelbar gegenüber“, sondern er muss ein „artifizielles Medium“ zwischen sich und die äußere Wirklichkeit einfügen. Er ist also darauf angewiesen, eine „Symbolwelt“ oder ein „Symbolnetz“ zu schaffen, um gleichsam „darin“ zu „leben“ und durch dieses zu kommunizieren. Diese den Menschen charakterisierende Fähigkeit und dessen Angewiesenheit auf Symbolisierung ist allerdings nicht das einzige Anthropologikum, also das entscheidende Kennzeichen des Menschen. Es handelt sich allenfalls um eine notwendige, aber keineswegs um eine hinreichende Bedingung. So setzen in vielfältiger Weise auch besonders Schimpansen und Bonobos, die uns genetisch sehr nahe stehen und auch z. B. Werkzeuggebrauch „erfunden“ haben, symbolische Gesten gezielt und situationsangepaßt ein. Sie können Symbolsprachen (wie die Taubstummensprache) in einer kleinen Lebens- und Lerngemeinschaft erlernen und sogar z. T. in kreativen Kombinationen verwenden. Sie vermögen taktisch gezielt zu täuschen und vorauszuplanen. Im übrigen benutzen Schimpansen sogar Werkzeuge, um andere Werkzeuge herzustellen, z.B. Anspitzen und Zurechtklopfen des spitzen Termitenbohrers mit dem stumpfen Bürstenende (in Kamerun von Sugiyama beobachtet). Und sie können den Werkzeugeinsatz im voraus planen ${ }^{1}$.

\footnotetext{
${ }^{1}$ So nahmen weibliche Schimpansen im westafrikanischen Urwald Steine zum Nüssezerschlagen mit, als sie zu meilenweiten Ausflügen in eine Zone aufbrachen, wo es wohlschmeckende, aber hartschalige Nüsse gab, jedoch keine Steine. Sie nahmen also vorausplanend Steine aus ihrem eigenen Habitat mit, um, da in diesem abgelegenen Gebiet keine Steine vorhanden waren, diese zum Nüssezerschlagen verwenden zu können! Dies wurde empirisch von dem Züricher Ehepaar Boesch durch viele Beobachtungen im Taï-Nationalpark bestätigt. Schimpansen benutzen sogar ganze Werkzeugsätze, u. U. verschiedene Werkzeuge für ein und dieselbe Tätigkeit oder ein einziges Werkzeug wie einen Stock für unterschiedliche Zwecke, etwa „als Riechsonde zur Erkundung, als Zahnstocher, als Angel zum Insektenfangen, als Bohrer zum Stochern nach Honig und Knochenmark, als Schaber zum Auskratzen von Nußschalen oder als Hebel zum Aufbrechen von Insektennestern“ bzw. Termitengängen, und „oft werden die Stöcke für den spezifischen Gebrauch besonders hergerichtet“. Neuerdings scheint sich gar herauszustellen, dass Schimpansen [in Kamerun, nach Sugiyama, zit. bei Peter Rene Becker, Werkzeuggebrauch im Tierreich (Stuttgart: Hirzelverlag, 1993), 102] ein Werkzeug (einen Stein) benutzen, um ein anderes Werkzeug, nämlich einen Stock zum Termitenangeln, herzustellen, indem sie die Ausfaserung und Ausfächerung am Stockende mit dem Steinhammer breitschlagen! [Jürgen Lethmate, „Vom Affen zum Halbgott. Die Besonderheiten des Menschen. Funkkolleg, Der Mensch - Anthropologie heute, Studieneinheit 2, Studienbrief 1 (Tübingen: Deutsches Institut für Fernstudien, 1992), 22f.]. Es gibt sogar zwei unterschiedliche „Werkzeugverhaltens-Zonen“ der Schimpansen: Das Nüsseknacken mit
} 
Entsprechendes gilt für die Entwicklung von Symbolgebräuchen und den Elementen einer sich traditional ausbreitenden Kultur, obwohl derartige Phänomene bei den Schimpansen natürlich nur in Ansätzen vorhanden sind und nicht systematisch in die Entwicklung eines konventionellen Symbolsystems eingebettet sind. Schimpansen können zwar in der Dressur eine Zeichensprache lernen und unter Umständen wie die Schimpansin Washoe (Gardner, Fouts) sogar von sich aus einem adoptierten Schimpansenkind beibringen, doch scheint sich bei ihnen die Eigenmotivation zur Entwicklung und Verwendung sowie spontanen und kreativen Weiterentwicklung von Symbolen einer systematischen darstellenden und benennenden Sprache nicht zu entwickeln. (Allerdings hatte Washoe gelegentlich ganz eigene Kombinationen von Zeichen, also eigene Sätze, „kreativ“ geäußert, indem sie etwa das Zeichen für „Hören“ anlässlich des Bellens eines Hundes mit dem Zeichen „Hund“ verband oder z.B. die Kombination „Bonbonbaum” für den Weihnachtsbaum einführte.) Schimpansen und Bonobos können darüber hinaus Eigenschaften und Relationen identifizieren, indem ein „,isoliertes Merkmal von seinem dinglichen Träger getrennt und seinerseits zum Verankerungspunkt in der symbolischen Identifikation gemacht wird“ (eine Art von symbolischer „Verdinglichung“), obwohl sie diese Fähigkeit im natürlichen Leben kaum benutzen. Es scheint, dass die „Schimpansensprache“ „ein Beiprodukt kognitiver Kategorien (ist), die eigentlich für eine andere Leistung benötigt werden “2.

Erst der Mensch hat die Fähigkeit, in systematischer Weise eine imaginäre Vorstellungsebene gleichsam zwischen sich und die Welt zu schieben und vielfältig neuartige Symbolkombinationen auf Kommunikationspartner zu übertragen. Dazu ist die sprachliche Symbolebene als weitere Stufe nötig, die eine formale

\footnotetext{
Steinen und Holzklötzen ist in vielen Nationalparks ganz im Westen Afrikas verbreitet, das Termitengraben mit einem dicken und kräftigen Grabstock etwa im heutigen Gabun und Kamerun und das Termitenfischen mit flexiblen dünnen Ästchen im westlichen Ostafrika, z. B. im berühmten Gombe-Nationalpark (ebd., 23, 56). Es gibt also eine Ausbreitung von Werkzeuggebrauchs- und Werkzeugherstellungskulturen bei Schimpansenstämmen, die offensichtlich doch in der Lage sind, entsprechend ihrem aktuellen Bedürfnis oder sogar antizipierend (!) Werkzeuge einzusetzen bzw. für spätere sozusagen ,geplante“ Tätigkeiten mitzunehmen. Weder Gebrauch noch Herstellung von Werkzeugen sind deshalb spezifisch für den Menschen. Und nicht einmal mehr der Gebrauch von Werkzeugen zur Werkzeugherstellung, was noch nach 1985 als ,spezifisch menschlich“ galt (Lethmate, Vom Affen zum Halbgott, 44). Allenfalls der Werkzeuggebrauch zur Herstellung von Werkzeugen zur Werkzeugherstellung - also die metastufliche Selbstanwendung - scheint doch „spezifisch menschlich“ zu bleiben.

${ }^{2}$ Norbert Bischof, Das Rätsel Ödipus: Die biologischen Wurzeln des Urkonflikts von Intimität und Autonomie (Piper: München/Zürich, 1985), 534f.
} 
Syntax, Repertoires von gemeinsamen Zeichen usw. voraussetzt. Er hat besonders die Fähigkeit, abstraktere interne Modelle eines zu imaginierenden Zusammenhangs von Zeichen, Eigenschaften und Beziehungen in zeitübergreifender Weise herzustellen. Die Tendenz, in klar abgegrenzten Modellunterscheidungen von Zeichen, Dingen, Ereignissen usw. zu operieren, „diese Tendenz, gewissermaßen in rechten Winkeln zu denken“, scheint doch erst „ein spezifischer Neuerwerb des Menschen“ $z$ u sein ${ }^{3}$. Nach Bischof ist die Schwierigkeit zur vorausschauenden „Vergegenwärtigung der Zeitachse“, also ein „neuer kognitiver Mechanismus (für) die Vergegenwärtigung nicht-gegenwärtiger Ereignisfolgen“, also die Fähigkeit „Zeitrepräsentation“ charakteristisch für die menschliche „Intelligenz“, wenn diese Fähigkeit nicht nur in Bezug auf die „gerade aktuelle Antriebslage“, sondern auch zur „Vergegenwärtigung künftiger Bedürfnislagen“ eingesetzt wird ${ }^{4}$. Jedoch reicht diese "richtige“ Vorwegvorstellung von späteren Bedürfnissen nicht zur klaren Abgrenzung des Menschen von den höchsten Primaten: Dies können wie erwähnt (Anm. 1) Schimpansenmütter in der Wildbahn auch leisten, wenn sie „vorausplanend” Stein- oder Holzhämmer“ zum Nüsseknacken mit sich tragen ${ }^{5}$. Somit reicht die vorstellende Vorwegnahme von späteren Bedürfnisentwicklungen, die „richtig vorausgesehen“ werden müssen, um die gegenwärtigen Antriebe danach auszurichten, also doch nicht als trennscharfes Kennzeichen des Menschen aus. Daher ist das antizipatorische „Antriebsmanagement“ also doch nicht allein das Charakteristische. So wird offenbar erst die auf der symbolischen höheren Ebene bezeichnete vorausschauende Bedürfniserfassung im Prozeß einer zeiteingebetteten Vergegenwärtigung künftiger Bedürfnisse und deren Erfordernisse zum wesentlichen Anthropikum und bedingt bzw. gestattet die Flexibilität seiner kulturellen symbolischen Erfindungen ${ }^{6}$.

Man kann wohl kaum bezweifeln, dass auch das Mitnehmen von „Werkzeug"-Steinen bei dem oben erwähnten Beispiel der Schimpansenwanderungen

${ }^{3}$ Ebd., 540.

${ }^{4}$ Ebd., $541 f$.

${ }^{5}$ Der Paläoanthropologe Mc Grew meint, allein der Mensch trage Nahrungsvorräte (Essbares, Wasser) in selbst hergestellten Beuteln mit sich. Dieses Anthropikon könnte man natürlich auch auf die in solchen oder ähnlichen Behältern mitgeführten Werkzeuge beziehen. So wäre der Mensch zwar nicht das einzige Werkzeuge herstellende (nicht einmal mit Hilfe von Werkzeugen, nach Sugiyarna, vgl. Anm. 1), sondern das künstliche Werkzeugkästen und oder -behälter transportierende Wesen!?

${ }^{6}$ Vgl. Bischof, Das Rätsel Ödipus, $540 \mathrm{ff}$. 
des Taï -Parks in ein anderes Gebiet schon der vorausschauenden und bedürfnisantizipierenden Bestimmung wenigstens ansatzweise gerecht wird ${ }^{7}$.

Wie steht es aber mit der symbolischen Erfassung oder Darstellung der künftigen Bedürfnissituation? Das „sprechende“ Bonobo-Genie Kanzi, das sogar einfaches Englisch versteht und durch Lexigrammtasten eines Bildcomputers durchaus aktiv kommuniziert, kann Handlungen - ähnlich wie die Schimpansin Washoe - recht weit voraus planen und sogar abwesende Situationsbedingungen vorgreiflich berücksichtigen ${ }^{8}$. Können Kanzi und Washoe sich jedoch auch äußern über die höherstufigen Zeichenbedeutung, also z.B. über die symbolischen Zeichenvarianten, mit denen sie künftig vorausgeahnte Bedürfnisse darstellen würden? Offenbar bleibt ihnen das ständige und systematische Höhersteigen auf Metastufen der Repräsentation verschlossen.

So dürfte die auf höheren Metastufen systematisch strukturierte Modellbildung in Gestalt von Zeichen für Symbole und Modellimaginationen zukünftiger Bedürfnislagen, also die metasprachliche symbolische Erfassung von Bedürfnisbeschreibungen in der vorausgreifenden Phantasie ein entscheidendes Abtrennungskriterium liefern.

Statt hier weitere inhaltlich differenzierende Charakteristika einzuführen, möchte ich eher methodologisch die Einsicht über die symbolische Stufung und die Zeichenebenen ernst nehmen und auf ein symbolisch-methodologisches Sonderphänomen aufmerksam machen, das in der Tat allein den Menschen kennzeichnen dürfte.

In Weiterführung von Cassirers eingangs erwähnter Auffassung des Menschen als des Animal symbolicum habe ich an anderer Stelle (1995) den Menschen als das metainterpretierende oder metasymbolische Wesen verstanden. Charakteristischerweise können Menschen - und wohl allein sie (außer vielleicht in engen Grenzen den „sprechenden Schimpansen” wie Washoe und Kanzi) - ihre Zeichen und die Symbolverwendung sowie die Symbolbildung auf einer höheren Metastufe wiederum zum Gegenstand von Symbolisierungsprozessen machen,

${ }^{7}$ Vgl. Christophe Boesch, Hedwige Boesch, „Dim Forest, Bright Chimps”, Natural History 1991, Nr 9: 50-57. Christophe Boesch, Hedwige Boesch, „Tool Use and Tool Making in Wild Chimpanzees", Folia Primatologica 1990, Nr 54: 86-99.

${ }^{8}$ Sue Savage-Rumbaugh, Stuat G. Shanker, Ttalbot J. Taylor, Apes, Language, and the Human Mind (Oxford 1998); Roger Fouts, Stephen Tukel Mills, Unsere nächsten Verwandten. Von Schimpansen lernen, was es heißt, ein Mensch zu sein (Limes Verlag: München, 1998). 
ihrerseits wieder der „Semiose” (Peirce) unterwerfen. Der Mensch ist also das Wesen, das in aufschichtender Höherstufung von Symbolen, Zeichen und deren Interpretationen einen symbolischen Aufstieg („symbolic ascent”) vornehmen kann, indem er Zeichenbildungen, Symbolisierungen, Interpretationen ihrerseits wieder als neue Objekte eines höherstufigen Symbolisierungsvorgangs entwerfen, erfassen und verwenden kann. Der Mensch kann Symbole wieder zum Gegenstand höherstufiger Symbole machen, Zeichen über Zeichen schichten, Symbole über Symbole konstruieren ${ }^{9}$, also nicht nur interpretieren, sondern gleichsam „metasymbolisieren” und gar „metainterpretieren”: Er verwendet Symbole für Symbole, interpretiert seine Interpretationen, ist also in diesem Sinne ein metasymbolisierendes Wesen - kurz: er ist das Animal metasymbolicum, das metainterpretierende Tier. Die Verwendung von ständig neuen Metaschichten setzt ein symbolisch-sprachliches Ergfassungsmittel auf den jeweils höheren Stufen voraus. Metaschichten lassen sich nur durch Metasprachbildung symbolisch erfassen, durchführen und durchgliedern. Das Metastufenwesen ist das Metasprachenwesen.

In der Tat scheint diese Fähigkeit wirklich charakteristisch für den Menschen zu sein. Auf dieser Möglichkeit einer stufenbildenden repräsentationalen Reflexion - im üblichen Sinne, aber auch als Rückbezüglichkeit („Rückbeugung“) gemeint - beruht die große begriffliche Variationsfähigkeit und Symbole verwendende Flexibilität des Menschen. Diese setzt nämlich die Fähigkeit voraus, höherstufige, also in der jeweiligen sprachlichen oder theoretischen Metastufe vollzogene Bezugnahmen auf Aktivitäten, Tätigkeiten - auch auf Strukturierungen derselben Art - vorzunehmen. Charakteristisch für das „Reflexivwerden der Mechanismen ${ }^{10}$ ist ja, dass wir das Organisieren organisieren, das Lernen lernen, das

\footnotetext{
${ }^{9} \mathrm{Vgl}$. Hans Lenk, Interpretationskonstrukte. Zur Kritik der interpretatorischen Vernunft (Frankfurt/Main: Suhrkamp, 1993), 22, 207 et passim; id., „Das metainterpretierende Wesen“, Allgemeine Zeitschrift für Philosophie 20.1 (1995): 39-47.

${ }^{10}$ Diese Tendenz zur Metastufenbildung bei Symbolisierungen und Interpretationen erinnert nicht nur an das „Reflexivwerden der Mechanismen“ bei Niklas Luhmann [Soziologische Aufklärung (Westdeutscher Verlag: Köln/Opladen 1970)], das terminologisch allerdings etwas irreführend so bezeichnet ist, insofern als die Stufenaufschichtung durch diese Selbstbezüglichkeit - eben von höherer Warte oder Ebene aus - nicht berücksichtigt wird. Und sie erinnert auch an das von Gregory Bateson [Natur und Geist (Suhrkamp: Frankfurt/Main, 1982), 146; Ökologie des Geistes: Anthropologische, psychologische, biologische und epistemologische Perspektiven (Suhrkamp: Frankfurt/Main, 1985, Orig. 1972), 244, 251, 287] betonte, bei ihm übrigens von Whorf
} 
Erkennen erkennen können und heute zunehmend auch müssen. (Es ist selbst moralisch geboten, moralischen Geboten zu folgen bzw. moralisch zu handeln.) Das alles kann nur durch Metastufenbildung geleistet und durch Metasprachenentwicklung beschrieben und weiterentwickelt werden.

Man denke im Anschluss an die produktive Fähigkeit des Menschen, neue Bedürfnisse zu generieren (was schon Karl Marx als charakteristisch für die menschlichen Gesellschaftszusammenhänge kannte), auch etwa an das Bedürfnis nach Bedürfnisbefriedigung bzw. -antizipation, das in diesem Sinne mit den Substitutionen von Metafähigkeiten für ursprüngliche Fähigkeiten des Menschen entsteht. (Entsprechend könnte auch sogenanntes „sekundäres”, also z.B. institutionelles Handeln als eine Abstraktform des spezifisch symbolgebundenen („symbolischen”) Metahandelns verstanden werden, bei dem Einzelne, eben als Repräsentanten einer Institution, quasi im sekundären Sinne handeln bzw. die Institution durch sie handelt, indem sie in stellvertretendem Sinne als Rollenträger repräsentative Handlungen individuell ausführen. Statt etwas schlicht zu leisten, um Bedürfnisse zu erfüllen, kann dem Mensch das Leisten selber wiederum zum Bedürfnis werden: Dem Eigenleistungswesen par excellence mag das Leistungsspiel zu einem l'art pour l'art werden.) Auch Bilder und Replays oder filmische Wiedergaben von Handlungen können gleichsam stellvertretend das ursprüngliche Bedürfnis nach Handlungen insofern überformen, als sie ein abgeleitetes, sekundäres, quasi metastufliches Bedürfnis erzeugen. Ein solchermaßen virtuelles Handeln bzw. „Bild(er)leben” wird in der Film- und Fernsehwelt oft geradezu zum Ersatz für das Bedürfnis nach wirklichem Handeln.

Der Mensch ist also nicht nur ein ,imitierender Generalist" darüber hinaus ein in Metastufen der Tätigkeitsbezüge transzendierender,

\footnotetext{
übernommene Modell der Metakommunikationen, Metafeststellungen, Metabehauptungen, die schon beim Spiel der Tiere vorhanden sind, indem diese etwa symbolisieren, dass es sich beim Kampfspiel nicht um Ernst handelt, sondern eben nur um ein nichtverletzendes Spiel. Dennoch dürfte es beim Menschen insofern anders sein, weil eben durch die Erfindung des Symbolsystems der Sprache und durch die Symbolisierung von Symbolen diese Metastufung nunmehr systematisch möglich ist.

${ }^{11}$ Andrew N. Meltzoff, Imitation, Objects, Tools and the Rudiments of Language in Human Ontology, zit. nach J. Lethmate, „Vom Affen zum Halbgott. Die Besonderheiten des Menschen“, 22
} 
metasymbolisierender und metainterpretierender sowie metahandelnder metasprachlicher Generalist: kurz ein Metastufen- und Metasprachenwesen ${ }^{12}$.

Wie an anderer Stelle diskutiert ${ }^{13}$ kann durch die Verweisungshandlung in der symbolischen Repräsentation von Ausführungshandlungen eine Unabhängigkeit von der gegenwärtigen Situation gewonnen werden. Dies macht die Bezugnahmen auf kontrafaktische, nichtbestehende Fakten und Prozesse möglich, gestattet Zeitenthobenheit und somit eine größere Flexibilität der Bezugnahmen auf Imaginäres. Es wurde gezeigt, dass man erst auf der Meta-Symbolebene eine große Variabilität und Flexibilität gewinnt. Die abstrakte, metasymbolische Bezugnahme auf nicht vorhandene Situationen, nicht-existierende Gegenstände, Sachverhalte usw. wird erst so möglich - und somit die Generalisierung auf bloß mögliche Konstellationen. Wir erreichen eine symbolische sprachliche (und zugleich stets metasprachliche) Repräsentation von Kontrafaktizität usw. Auch dies ist natürlich gebunden an die Möglichkeit, von der Einführungsphase einer Nachahmung oder direkten Wahrnehmung aufzusteigen zu der durch Symbolisierung und Anführungen bzw. repräsentierende Stellvertretungen ermöglichten $\mathrm{Me}$ taebene. Es handelt sich um den Prozeß des Abstrahierens, der durch den Übergang in Metastufen der Anführung bzw. Bezugnahme und Symbolverwendung konstituiert wird. Symbolische Darstellungen aller Arten und höherer Stufen, also auch metasprachliche Strukturen sind notwendig an diese Aufschichtung gebunden. Ohne differenziertere Metasprachebenen und -formen lassen sich diese gar nicht durchführen, geschweige denn, genauer erfassen oder beschreiben. Diese in systematischer Weise zu vollziehen und auch im kulturellen Zusammenhang

${ }^{12}$ All dies hängt natürlich damit zusammen, dass durch die Entwicklung von Symbolsystemen und durch die Einführung konventioneller Symbole in bestimmten Lernphasen und aufgrund einer funktionalistischen Gebrauchstheorie der Symbolbedeutungen Handlungen durch Symbole vertreten und so als Gegenstände dargestellt werden können - sei es durch bildliche Wiedergabe wie im Foto oder Film, durch Zeichnungen oder Diagramme, sei es durch abstrakte nicht-ikonische Symbole, durch Imitation, generalisierende Stilisierung von Strukturen usw. Durch die Ausführung von symbolischen Anführungshandlungen für echte Grundhandlungen (durch eine filmische Wiedergabe von einer erotischen Handlung) lässt sich z.B. eine (erotische) Emotion beim Zuschauer erzeugen), kann sozusagen die Repräsentationshandlung die Aktualisierung der Ursprungshandlung vorspiegeln, imaginativ reaktivieren usw. Entsprechendes gilt natürlich bei antizipatorischen Vorstellungen für künftige bedürfnisbefriedigende Handlungen oder gar Bedürfnislagen, höherstufige Wünsche (Volitionen zweiter Ordnung nach Harry Frankfurt).

${ }^{13}$ Lenk, Interpretationskonstrukte, $469 \mathrm{ff}$. 
gemeinsam zu vollziehen und auf andere Kommunikationspartner „übertragen” (mittels des gemeinsamen Repertoires und der entsprechenden Symbolgrammatik in hinreichend ähnlicher Weise anklingen lassen) zu können - das ist charakteristisch für dieses Metastufenkonzept beim menschlichen Benennen, Erkennen, Kommunizieren und auch beim Handeln mittels symbolischer Repräsentationen und auf höheren symbolisch vermittelten Metastufen.

Zusammenfassend kann man sagen, dass der Mensch nicht nur das metasymbolische und metahandelnde Wesen ist, das Symbolisierungen für Symbole verwendet und durch Symbolverwendung handelt, sondern er ist auch das Metasprachenwesen. Er ist bei seinen Repräsentationen und in allen Weisen der sprachlichen Erfassung und Beschreibung fähig, auf Metasprachstufen und immer höhere Aufschichtungen zurückzugreifen ${ }^{14}$. Der Mensch ist das Lebewesen, das generell der Selbstbezugnahme auf der Metastufe fähig ist, das stets zu abstrakteren, höheren Metastufen der sprachlichen oder symbolischen Darstellung (Metasprache) oder der theoretischen Erfassung (Metatheorie) aufzusteigen vermag. Er ist aber nicht nur das metarepräsentierende, sondern auch das metahandelnde Wesen (stellvertretendes, institutionelles, sekundäres Handeln in bezug auf das zugrundegelegte Handeln), des Interpretierens des Interpretierens, des Symbolisierens des Symbolisierens usw. fähig. Er ist das Metasprachwesen par excellence.

\section{Literatur}

Bateson, Gregory. Natur und Geist. Suhrkamp: Frankfurt/Main, 1982.

Bateson, Gregory. Ökologie des Geistes: Anthropologische, psychologische, biologische und epistemologische Perspektiven. Suhrkamp: Frankfurt/Main, 1985 (Orig. 1972).

Becker, Peter Rene. Werkzeuggebrauch im Tierreich. Hierzel Verlag: Stuttgart, 1993.

Bischof, Norbert. Das Rätsel Ödipus: Die biologischen Wurzeln des Urkonflikts von Intimität und Autonomie. Piper: München/Zürich, 1985.

Bochenski, I.M. Ancient Formal Logic. North-Holland Publishing: Amsterdam 1951.

Bochenski, I.M.. Formale Logik. Karl Alber Verlag: Freiburg - München, 1956.

${ }^{14}$ Theoretisch mögen die Aufschichtungen unbegrenzt sein, praktisch endet das Höhersteigen in der aktualisierten mentalen Vorstellung recht bald: Wir haben schnell Abstraktionsschwierigkeiten, z. B. die Metaschichten mental zu trennen oder oft schon klar zwischen der bezeichnenden Vorstellung und dem bezeichneten Begriff oder Gegenstand zu unterscheiden. 
Boesch, Christophe, Hedwige Boesch. „Dim Forest, Bright Chimps”. Natural History 1991, Nr 9: 50-57.

Boesch, Christophe, Hedwige Boesch. „Tool Use and Tool Making in Wild Chimpanzees”. Folia Primatologica 1990, Nr 54: 86-99.

Cassirer, Ernst. Versuch über den Menschen. S. Fischer Verlag: Frankfurt .1990 (Orig. 1944).

Chi, R. S.Y. Buddhist Formal Logic I: A Study of Dignaga's Hetucakra and K'uei-Chi's Great Commentary on the Nyayapravesa. Delhi 1984 (Orig. 1969).

Fouts, Roger, Stephen Tukel Mills, Unsere nächsten Verwandten. Von Schimpansen lernen, was es heißt, ein Mensch zu sein. München: Limes Verlag, 1998.

Graham, Angus Charles., Later Mohist Logic. Ethics and Science. Hongkong-London 1978.

Harbsmeier, Christoph. „Chinese Buddhist Logic“. In: Joseph Needham (Hg.), Science and Civilisation in China, 358-409. Bd.7, Teil I: Language and Logic. Cambridge UP: Cambridge, 1998.

Lenk, Hans, Rainer Hegselmann. „Partikeln, logische (Begründung der 1.P.)“. In: Historisches Wörterbuch der Philosophie, Band 7. Schwabe AG: Basel, 1989.

Lenk, Hans, Gregor Paul (Hg.). Epistemological Issues in Classical Chinese Philosophy Albany. State University of New York Press: NY, 1993.

Lenk, Hans. Kritik der logischen Konstanten, De Gruyter: Berlin 1968.

Lenk, Hans. „Logik, Cheng Ming und Interpretationskonstrukte“, Zeitschrift für philosophische Forschung 45 (1991): 391-401.

Lenk, Hans. Metalogik und Sprachanalyse. Verlag Rombach: Freiburg, 1973.

Lenk, Hans. „Philosophische Logikbegründung und rationaler Kritizismus“, Zeitschrift für philosophische Forschung 24 (1970): 183-205 (wieder abgedruckt in ders., 1973, 88-109).

Lenk, Hans. „Zur Frage der apriorischen Begründbarkeit und Kennzeichnung der logischen Partikeln“. In: Carl Friedrich Gethmann, Logik und Pragmatik, 11-35. Suhrkamp: Frankfurt/Main, 1982.

Lenk, Hans. „Das metainterpretierende Wesen“. Allgemeine Zeitschrift für Philosophie 20.1 (1995): 39-47.

Lenk, Hans. Interpretationskonstrukte: Zur Kritik der interpretatorischen Vernunft. Suhrkamp: Frankfurt/Main, 1993.

Lethmate, Jürgen. „Evolutionsökologie der Menschenähnlichen (Homenoidea)“. Praxis der Naturwissenschaften/Biologie 38 (1) (1989): 7-15.

Lethmate, Jürgen. Vom Affen zum Halbgott. Die Besonderheiten des Menschen. Funkkolleg „Der Mensch - Anthropologie heute” (Studieneinheit 2, Studienbrief 1). Tübingen 1992; 60-76).

Lindtner, Ch., „Madhyamaka Causality“. Horin 6 (1999).

Lorenz, Konrad. Indische Denker. Verlag C.H. Beck: München, 1998.

Lorenzen, Paul. Einführung in die operative Logik und Mathematik. Springer: Heidelberg-Göttingen-Berlin, 1955.

Luhmann, Niklas. Soziologische Aufklärung. Westdeutscher Verlag: Köln/Opladen, 1970. 
Mauthner, Fritz. Beiträge zu einer Kritik der Sprache. 3 Bde. Ullstein: Frankfurt/Main.-BerlinWien, 1982 (Orig. 1901, 1906²).

Meltzoff, A. N. „Imitation, Objects, Tools and the Rudiments of Language in Human Ontology:“. Human Evolution 3 (1988): 45-64.

Oetke, Claus. Studies on the Doctrine of Trairupya, Arbeitskreis für Tibetische und Buddhistische Studien Universität Wien: Wien, 1994.

Paul, Gregor. „Argumente für die Universalität der Logik. Mit einer Darstellung äquivalenter Axiome Aristotelischer Syllogistik, spätmohistischer Logik und buddhistischer Begründungstheorie“. Horin. Vergleichende Studien zur japanischen Kultur 1,1 (1994): 57-86.

Paul, Gregor. Die Aktualität der klassischen chinesischen Philosophie. Iudicum Verlag: München, 1987.

Renou, Luis. „Sur le genre du sutra dans la littérature sanskrit“. Journal Asiatique 251 (1963): 165-216.

Savage-Rumbaugh, Sue, Stuart G. Shanker, Talbot J. Taylor. Apes, Language, and the Human Mind. Oxford University Press: Oxford, 1998

Scharfe, Hartmut. Panini's Metalanguage. American Philosophical Society: Philadelphia, 1971.

Scherer, Bernd Michael. Prolegomena zu einer einheitlichen Zeichentheorie. Ch. S. Peirce'Einbettung der Semiotik in die Pragmatik. Stauffenburg Verlag: Tübingen, 1984.

Schiefenhövel, Wulf, Christian Vogel, Gerhard Vollmer. Von der Wiege bis zur Bahre: Was uns am Menschen interessiert. Funkkolleg „Der Mensch: Anthropologie heute”. Studieneinheit 1. Studienbrief 1. Tübingen: Deutsches Institut für Fernstudien 1992: 1-64.

Scholz, Heinrich. Metaphysik als strenge Wissenschaft. Staufen: Köln, 1941.

Sommer, Volker. Lob der Lüge: Täuschung und Selbstbetrug bei Tier und Mensch. Beck: München, 1992.

Staal, Frits. Rules without Meaning: Ritual, Mantras in the Human Sciences. State University of New York: New York, 1988.

Staal, Frits. „The Concept of Metalanguage and its Indian Background“, Journal of Indian Philosophy3 (1975): 315-354.

Staal, Frits. Universals: Studies in Indian Logic and Linguistics, University of Chicago Press: Chicago-London, 1988.

Tachikawa, Musachi. „A Sixth-Century Manual of Indian Logic (A Translation of Nyayapravesa)“. Journal of Indian Philosophy 1 (1971): 111-145.

Trivers, Robert L. „The Evolution of Reciprocal Altruism“. Quarterly Review of Biology 46 (1971): 35-57. 


\section{Summary}

\section{The meta-level being is the meta-language being}

Starting from Cassierer's idea as the human as the symbolic being (animal symbolicum) I specify and interpret humans as the meta-symbolic, i.e. meta-interpreting beings. In principle, they can always go up to higher and higher levels of language (meta-language) and interpretation (meta-interpretation). That is an anthropological characteristic property of humankind.

Keywords: Meta-interpreting being, animal symbolicum et meta-symbolicum, meta-language, meta-levels, anthropologicum

\section{Streszczenie}

\section{Istota meta-poziomowa jest istotą meta-językową}

Wychodząc od koncepcji Cassirera, zgodnie z którą człowiek jest istotą symboliczną (animal symbolicum), określam i interpretuję ludzi jako istoty meta-symboliczne, tj. jako istoty meta-interpretujące. W zasadzie, mogą oni zawsze wchodzić na coraz to wyższe poziomy języka (meta-języka) i interpretacji (meta-interpretacji). Jest to antropologiczna charakterystyka właściwości cechującej gatunek ludzki.

Słowa kluczowe; istota meta-interpretujaca, animal symbolicum et meta-symbolicum, meta-język, meta-poziomy, anthropologicum, kryterium człowieczeństwa

Informacje o autorze:

HANS LENK, profesor emerytowany, Karlsruhe Institute of Technology, Germany; e-mail: hans.lenk@kit.edu

\section{(cc) BY-NC-ND}

